\title{
EXPLORANDO LA PARTICIPACIÓN OCUPACIONAL EN NIÑOS CON EPIDERMÓLISIS BULLOSA INTEGRADOS AL SISTEMA EDUCACIONAL CHILENO
}

\author{
EXPLORING TO OCCUPATIONAL PARTICIPATION IN CHILDREN WITH EPIDERMOLISYS \\ BULLOSA INTEGRATED INTO THE CHILEAN EDUCATIONAL SYSTEM
}

\begin{abstract}
Javiera Aubert V., Lorena Berenguer M., María Jesús Cofré D., Catalina Leyton A. , Alejandra González M. ${ }^{2}$, Rodrigo Sepúlveda $\mathbf{P} .^{3}$
\end{abstract}

\section{Resumen}

El presente estudio explora la participación ocupacional en el área de educación de niños con Epidermólisis Bullosa (E.B.) en sus tipos distrófica y de la unión, los cuales, dada su condición de salud general son considerados como estudiantes con necesidades educativas especiales (NEE).

Con el objetivo de conocer si la integración es satisfactoria y cuenta con los apoyos necesarios, se realiza mediante un estudio de tipo exploratorio cualitativo, un análisis de tres casos según criterios de inclusión establecidos. A través de un análisis pragmático de los datos obtenidos durante el trabajo de campo se realiza el perfil ocupacional inicial del niño (S.C.O.P.E.), instrumento propio del Modelo de Ocupación Humana, desprendiéndose de éste conclusiones, que se estructuran en base al mismo modelo.

La importancia y relevancia de estudiar esta temática está dada por la pertinencia de la intervención y el aporte que la Terapia Ocupacional puede brindar a las personas con E.B., que si bien desde el punto de vista epidemiológico tiene una baja incidencia en la población general, quienes la padecen requieren de un abordaje multidisciplinar e integral, dado por la complejidad de la condición de salud y el impacto que esta provoca en todas las áreas de la ocupación.

\section{Palabras clave:}

Epidermólisis Bullosa, Necesidades Educativas Especiales, Participación Ocupacional, Modelo de Ocupación Humana, Terapia Ocupacional.

\begin{abstract}
This study explores the occupational participation of children with Epydermolisys Bullosa (E.B.) dystrophic and junctional in the educational area, which because of their health condition, are considered as student with Special Educational Needs (SEN).

With the aim to know how satisfactory is the integration and whether it has the necessary support, the study carried out, through a qualitative and exploratory focus, on three selected cases with the established selection criteria. Through a pragmatic analysis of data obtained during the field work, the Short Child Occupational Self Assessment (S.C.O.P.E.) is used, from the Model of Human Occupation (M.O.H.O.), some conclusions emerge, which are structured and based on the model announced.
\end{abstract}

The importance and relevance of studying this subject from Occupational Therapy is given by the pertinence of the intervention and contribution to E.B. in people who live this condition, even though it as a low incidence in general population from an epidemiological perspective, because they need a multidisciplinary and integral intervention to mitigate the impact caused by the disease in all occupational areas.

\section{Keywords:}

Epidermolisys Bullosa, Special Educational Needs, Occupational Participation, Model of Human Occupational, Occupational Therapy.

\footnotetext{
1 Terapeuta Ocupacional, Universidad de Chile.

2 Terapeuta Ocupacional, Universidad de Chile. Magister en Administración y Gestión en Salud, Universidad de los Andes.Docente Escuela de Terapia Ocupacional, Universidad de Chile agonzalezmoreno@med.uchile.cl

${ }^{3}$ Antropólogo, Académico Escuela de Terapia Ocupacional, Universidad de Chile.
} 


\section{INTRODUCCIÓN}

La Epidermólisis Bullosa (E.B.), es un grupo de patologías hereditarias, caracterizada por la tendencia a formar ampollas, úlceras y heridas en la piel y las mucosas, ante el mínimo traumatismo o golpe (Solaque, H. y Vergara, E. 2009). Impacta en algunos casos el funcionamiento de otros órganos, sin afectar directamente el área cognitiva de quienes la padecen. Los datos epidemiológicos a nivel mundial corresponden a una prevalencia de 8,22 pacientes con E.B. por 1.000 .000 de habitantes y una incidencia de 19,6 nacidos con E.B. por 1.000 .000 de nacidos vivos, afectando en igual porcentaje a hombres y mujeres (Fine, J. 2010).

En Chile, la prevalencia es de 10 personas con E.B. por 1.000.000 de habitantes. La distribución corresponde a: $57 \%$ de tipo simple, $38 \%$ es distrófica y $5 \%$ corresponde a de la unión. La mayor cantidad de casos se encuentran en la Región Metropolitana, donde se presenta un total de 62 casos; seguida por la VI Región con un total de 25 casos. En cuanto a la distribución por rango etário, un $48 \%$ de los usuarios son menores de 10 años, un $33 \%$ se encuentra entre los 10 y 20 años y un $19 \%$ son mayores de 20 años (Yubero, M. et al. 2010).

Si bien la cantidad de casos en el país no sobrepasa los 200, es importante abordar el impacto que tiene esta enfermedad en la calidad de vida de las personas con EB y principalmente los aspectos ocupacionales que pueden verse involucrados. Este estudio se enfoca específicamente en usuarios diagnosticados con E.B. de los tipos distrófica y de la Unión, debido a su alto compromiso multisistémico, y por consiguiente la afección en la participación ocupacional, entendida como el desempeño satisfactorio en las diversas áreas de la ocupación (descanso y sueño; actividades de la vida diaria básicas e instrumentales; educación, trabajo; participación social; ocio y tiempo libre) según el contexto sociocultural propio, que son deseadas o necesarias para el bienestar personal (Kielhofner, G. 2006).

Las diversas formas de presentación de la E.B. se pueden agrupar en tres tipos principales según sus ca- racterísticas histológicas: simple, que presenta ampollas intraepidérmicas, localizadas a nivel de la capa basal de la piel, siendo éste el tipo menos grave; distrófica, que presenta ampollas a nivel de la dermis y se manifiesta de manera severa si ésta se expresa a través de herencia recesiva, o de manera más leve si lo hace a través de la herencia dominante; y de la unión, que presenta ampollas a nivel de la membrana basal de la unión dermoepidérmica y es la forma menos frecuente de presentación, de herencia recesiva y con manifestaciones que pueden llevar a la persona a la muerte en los primeros años de vida (3). Esta patología es de carácter crónico y no varía de un tipo a otro.

Los tipos distrófico y de la unión son los más severos. Impactan de manera global a la persona, afectando además de la piel a otros tejidos y órganos. Dependiendo del subtipo, la E.B. puede provocar afecciones como pseudosindáctila en manos y pies, deformidades, afección de la mucosa oral o esmalte dental, desnutrición progresiva y anemia, entre otras. Estas afecciones impactan tanto en la persona como en su entorno, restringiendo a menudo su participación ocupacional. El desempeño ocupacional, entendido como un proceso dinámico que surge del resultado de una transacción constante entre la persona, el ambiente y la ocupación, se puede ver interferido en el caso de las personas con E.B. por las características de la misma, las demandas ocupacionales, las barreras físicas, sociales y culturales (Blesedell, E. et al. 2005).

El estudio considera a los niños que asisten al primer ciclo de educación escolar, el cual abarca de $1^{\circ}$ a $8^{\circ}$ básico. Este periodo es fundamental para la adquisición de conocimientos que tienen como finalidad proporcionar a todos los estudiantes una formación común que haga posible el desarrollo de las capacidades individuales motrices, de equilibrio personal, de relación y de actuar social. El rango etario considerado en el estudio es entre 6 y 16 años de edad, que corresponde al $48 \%$ de usuarios con E.B. registrados por la Fundación DEBRA Chile ${ }^{3}$ en sus tipos más severos.

Durante el transcurso de la educación básica se desarrollan habilidades educativas necesarias para los siguientes niveles escolares y el resto de la vida, siendo el ambiente escolar un potenciador del desarrollo integral del niño. En cuanto al desarrollo cognitivo, según

3 Fundación Debra Chile: Entidad sin fines de lucro, que brinda apoyo, educación y atención médica a todos los pacientes chilenos portadores de Epidermólisis Bullosa. 
Piaget (1936), una vez finalizado el ciclo escolar se espera que el niño alcance un pensamiento abstracto que le permitirá emplear un pensamiento lógico, inductivo y deductivo necesario para desarrollar sus habilidades como persona.

La importancia de la asistencia de los niños al sistema educacional, radica entre otros, en la dinámica escolar que se genera, que facilita el desarrollo moral, mediante la relación que el niño establece con sus compañeros y figuras de autoridad. Es esperado que durante esta etapa se transite desde la heteronomía hacia la autonomía, en la cual debe ser capaz de aplicar las normas y conceptos morales de acuerdo a sus propios principios y valores (Kohlberg, 1958). Simultáneamente, en cuanto al desarrollo afectivo, los estudiantes en esta etapa se encuentran en el estadio de laboriosidad vs. inferioridad, en el cual se desarrolla el proceso de aprendizaje de las destrezas necesarias para la vida, lo que refuerza el autoconcepto y la autoestima. Al finalizar la etapa escolar básica, el estudiante se encuentra en el estadio de identidad vs. confusión de identidad, donde se espera que comience a formar su identidad, organizando sus habilidades, necesidades y deseos para adaptarlos a las exigencias de la sociedad (Erikson, 1983).

Debido a las características de la E.B. en sus tipos más severos, este grupo de niños y jóvenes se consideran como alumnos(as) con necesidades educativas especiales (N.E.E.), dado que requieren ayudas y recursos adicionales, ya sean humanos, materiales o pedagógicos para conducir su proceso de desarrollo y aprendizaje, y contribuir al logro de los fines de la educación.

La Ley 20.422, promulgada por el Gobierno de Chile el año 2010, establece las normas sobre igualdad de oportunidades de inclusión social de personas con discapacidad, definiéndose ésta como "la ausencia de discriminación por razón de discapacidad, así como la adopción de medidas de acción positiva orientadas a evitar o compensar las desventajas de una persona con discapacidad para participar plenamente en la vida política, educacional, laboral, económica, cultural y social" (Ley N²0.422, 2010). Para favorecer la inclusión educacional, el Gobierno ha implementado diversos planes, dentro de los cuales se encuentra el de integración educacional.

La integración educacional corresponde a la participación del alumno(a) con N.E.E. en el aula regular, lo cual le permite vincularse a las actividades académicas y extra académicas, favoreciendo el proceso de aprendizaje y el desarrollo de habilidades de sociabilización, necesarias para un desempeño apropiado en la comunidad (Rojas, X. 2008). Para lograr la adecuada integración del estudiante con E.B. en sus tipos más severos al aula, existen estrategias tales como realizar innovaciones y adecuaciones curriculares, de infraestructura y proporcionar los materiales de apoyo necesarios.

El modelo que guía la presente investigación es el Modelo de Ocupación Humana, el cual comprende al ser humano como un sistema abierto, formado por tres elementos principales, la volición (causalidad personal, valores e intereses), es decir la motivación hacia las ocupaciones; la habituación (hábitos y roles), forma única en la cual cada persona organiza sus ocupaciones; y la capacidad de desempeño, que corresponde a la utilización de los componentes físicos y mentales, sumado a la experiencia para realizar dichas ocupaciones. Como resultado de la interacción constante entre los componentes de la persona con el ambiente, ya sea éste físico o social, se produce el hacer, que en su dimensión más básica se traduce en las habilidades motoras, de procesamiento y de comunicación e interacción, las cuales se expresan en el desempeño ocupacional, es decir la realización de una tarea. El desempeño en diversas ocupaciones genera la participación ocupacional. Con el tiempo la participación del individuo en el trabajo, juego y actividades de la vida diaria, forman y mantienen la identidad ocupacional y la competencia ocupacional, las cuales construyen en conjunto la adaptación ocupacional. Debido a las características de la E.B. en sus tipos más severos, el alto compromiso físico, impacto psicológico y la demandas que esto implica para el usuario y su familia, se puede ver interferido cualquiera de los componentes anteriormente señalados, repercutiendo en la participación ocupacional del usuario en el área de la educación, y por consiguiente en su adaptación ocupacional (Kielhofner, G.2006).

Dado lo anterior, el objetivo de este estudio se orienta a explorar las necesidades, facilitadores y obstáculos que enfrentan los usuarios, sus familias y las instituciones educativas, constituyendo éstos aspectos claves para resolver la siguiente pregunta: ¿Cómo es la participación ocupacional de personas con Epidermólisis Bullosa distrófica y de la unión integrados en el sistema educacional chileno?

Para responder esta interrogante, durante el año 2012 se realiza un estudio de carácter exploratorio que se basa en el análisis de tres casos escogidos de un total de 24 usuarios con E.B., con edades entre 6 y 16 años, beneficiarios de la Fundación Debra Chile, y asistiendo a la educación básica en el sistema de educación formal. 


\section{Metodología}

El presente estudio tiene un enfoque cualitativo, que se refiere "en su más amplio sentido a la investigación que produce datos descriptivos: las propias palabras de las personas, habladas o escritas, y la conducta observable" (Taylor, S. J. \& Bogdan, R. 1987). A la vez el estudio es de tipo exploratorio debido a que existe escasa información sobre el tema y a través de ésta se espera generar conocimientos para el desarrollo de nuevas investigaciones.
El universo de estudio está conformado por usuarios con diagnóstico de E.B. de tipo distrófica y de la unión en Chile. Con el propósito de dar respuesta a la pregunta de investigación, se realizan tres estudios de caso, que además considera a los cuidadores principales y profesores jefes.

Se seleccionan tres usuarios (ver tabla 1) para la realización de los estudios de caso, intentando profundizar en todos los aspectos que influyen en la participación ocupacional en el área de educación.

Tabla 1: Participantes seleccionados

\begin{tabular}{|c|c|c|c|c|c|c|c|}
\hline Caso & Nombre & Edad & Sexo & Región & $\begin{array}{c}\text { Tipo de } \\
\text { E.B. }\end{array}$ & $\begin{array}{c}\text { Tipo de } \\
\text { educación }\end{array}$ & Curso \\
\hline 1 & V.V. & 15 años & M & R.M. & Distrófica & Especial & Nivel 10 \\
\hline 2 & C.S. & 7 años & F & VII & $\begin{array}{c}\text { De la } \\
\text { unión }\end{array}$ & Regular & $1^{\circ}$ básico \\
\hline 3 & C.H. & 14 años & M & VII & Distrófica & Regular & $3^{\circ}$ básico \\
\hline
\end{tabular}

Los participantes se seleccionan de la base de datos de la Fundación Debra Chile, según la accesibilidad para realizar la investigación y cumplen con los siguientes criterios de inclusión:

- Asistir al ciclo de educación básica.

- Tener edad entre 6 y 16 años.

El instrumento de evaluación utilizado es el perfil ocupacional inicial del niño (S.C.O.P.E.) propio del Modelo de Ocupación Humana. Provee una visión amplia de la participación ocupacional de usuarios menores de 21 años, dado que permite evaluar los factores que reafirman o limitan la participación ocupacional, siendo éstos propios del niño (volición, habituación y capacidad de desempeño) o del ambiente (físico o social) en el cual se desempeña. Cabe destacar que este instrumento evalúa a la persona en comparación con su propia trayectoria individual de desarrollo y no con un conjunto de estándares preestablecidos, siendo estos argumentos válidos para considerarlo como el instrumento más pertinente para analizar los elementos necesarios, para dar respuesta a la pregunta de investigación.

El instrumento permite flexibilizar la manera de obtener la información necesaria, por lo que se crean cinco pautas: una de observación del ambiente escolar, una de observación de las habilidades del usuario y tres de entrevistas semi estructuradas, que son aplicadas al niño o joven, al cuidador principal y al profesor jefe. Estas pautas se cotejan, con el objetivo de comprobar su eficacia, en tres casos de personas sin discapacidad, con edades entre 6 y 16 años, que asisten a la educación formal. Las cinco pautas fueron aplicadas a cada caso, en sus colegios y en el hogar, para luego complementarse entre sí y obtener las conclusiones que arroja el estudio.

El estudio considera aspectos éticos, a través de la mantención de la confidencialidad de los participantes, mediante la omisión de nombres y el uso de siglas. La participación de las personas es voluntaria, y se les entrega información oportuna sobre los propósitos, metodología y beneficios del estudio. Se aplica un consentimiento informado a todos los participantes mayores de edad, y un asentimiento informado a los participantes menores de edad.

\section{Análisis de Resultados}

Se realiza el análisis del perfil ocupacional inicial de cada participante a través de los resultados obteni- 
dos en las pautas de observación y entrevistas semi estructuradas. Dicho proceso se lleva a cabo mediante un enfoque de análisis pragmático, en el cual se analizan los procesos prácticos y personales que intervienen en éste, considerando diferentes variables relevantes para la comprensión de un caso o para explicar la elección de determinadas formas de realizar el enunciado en función de los factores contextuales (1). Se analizan frases textuales de las entrevistas y notas de campo obtenidas durante el proceso en terreno, para luego identificar aspectos que se asemejan a los criterios planteados para clasificar cada ítem según si éstos reafirman ("Facilita", "Permite") o limitan ("Restringe" o "Inhibe") la participación ocupacional de dicha persona, permitiendo identificar aquellas áreas que son consideradas fortalezas y desafíos en su contexto educacional.

\section{CASO 1:}

\section{- Contexto}

V.V. es un joven de 15 años y diagnóstico de E.B. distrófica, es hijo único y vive con sus padres en MelipiIla (RM). El presente año se encuentra asistiendo a una escuela especial donde cursa el nivel 10. Cabe destacar que previo a su incorporación a esta escuela, V.V. se encontraba estudiando en un establecimiento educacional regular, del cual tuvo que retirarse debido a que sufría de bulling de parte de sus compañeros y no logró adquirir habilidades de lectoescritura y cálculo básico acorde a su edad.

\section{- Análisis del Perfil Ocupacional}

\section{Fortalezas:}

El joven se ha adaptado adecuadamente al contexto escolar, siendo capaz de involucrarse activamente en las rutinas y adquirir hábitos que facilitan su incorporación; su profesora jefe refiere: "No ha necesitado apoyo para adaptarse a las rutinas escolares, comprende muy bien, es muy activo". Por otro lado, V.V. posee una adecuada expresión verbal, lo cual facilita su proceso de incorporación en el nuevo contexto escolar, a pesar de los problemas de articulación de las palabras, que no afecta la interacción con sus compañeros y el resto de la comunidad escolar, reflejándose en la siguiente cita de la profesora: "El lenguaje es atingente a su edad, no tiene dificultades para iniciar o mantener conversaciones, sólo presenta dificultades, en ocasiones, por el tema de la articulación de las palabras".

Desafíos:

En cuanto a la volición, el joven posee una adecuada exploración del ambiente, pero se ve interferido por su inseguridad, que se evidencia en la continua búsqueda de aprobación familiar, quienes en ocasiones lo sobreexigen y en otras lo sobreprotegen, produciendo en él una actitud autoexigente; la madre refiere: "tú le colocas la tarea, se la explicas y él al tiro, en 5 segundos ya la tiene lista, lo que él más busca es que le des más y más tareas para seguir avanzando, es muy trabajólico, yo le tengo que estar diciendo que haga su trabajo más lento y pausado". Por otro lado, el joven posee buenas habilidades cognitivas y de comunicación e interacción, en este sentido la profesora jefe refiere: "yo lo trato igual que al resto de sus compañeros, en lo cognitivo todos igual, pero a diferencia de sus compañeros trabaja más rápido y comprende bien".

Las habilidades motoras del usuario se ven restringidas debido a la manifestación de la patología, las cuales en su caso se han expresado principalmente en pseudosindactilia (manos en capullo), rigidez producto de las cicatrices y múltiples heridas, lo que limita su posibilidad de exploración autónoma de su propio entorno. V.V. refiere: "a veces voy al colegio en silla de ruedas, por las heridas en los pies, tengo que pedir ayuda para moverla". Estos aspectos limitan la capacidad de realización de actividades gruesas y finas, sumado a la disminución en el nivel de energía durante las actividades, su profesora relata: "en el día a día se cansa y eso mismo le hace bajar el ánimo, al final de la jornada tiene dificultades para realizar las actividades académicas".

Además, los recursos físicos del ambiente escolar son insuficientes, no existen adaptaciones ni ayudas técnicas que suplan dichas limitaciones, lo cual restringe la participación ocupacional del joven, su madre refiere: "dentro de la sala de clases no tienen adaptaciones y fuera de ella tampoco".

Respecto al ambiente social de la escuela, los miembros de ésta se encuentran sensibilizados acerca de la condición de V.V., sin embargo muestran una actitud de sobreprotección generalizada, que se observa en una marcada restricción a participar en actividades sociales que no evidencian un riesgo potencial, y las escasas demandas ocupacionales a las que el joven se ve enfrentado dentro de este contexto; su profesora jefe afir- 
ma: "Su jornada es diferente, pierde las horas de taller, pierde el taller de psicomotricidad, repostería, deporte y cálculo lúdico, los temas más complicados para él... durante el recreo espera que sus compañeros de clases salgan a recreo y se va a la oficina de la directora, así evitamos golpes y accidentes que puedan ocurrir en el recreo".

\section{CASO 2:}

\section{- Contexto}

C.S. es una niña de 7 años diagnosticada con E.B. de la unión, quien reside con sus padres en la ciudad de Talca (VII Región). Desde hace 4 años asiste a una escuela rural y actualmente se encuentra en $1^{\circ}$ básico. Debido a las características de su tipo de E.B., C.S. ha estado hospitalizada en reiteradas ocasiones durante el año escolar en curso, por lo cual su asistencia ha sido baja.

\section{- Análisis del Perfil Ocupacional}

\section{Fortalezas:}

La menor posee un desarrollo cognitivo que favorece su inclusión escolar, lo cual se evidencia en su capacidad de comprender, identificar, organizar y utilizar de manera adecuada los objetos que se le presentan cotidianamente en dicho contexto, lo que es influenciado por el ambiente social en el cual está inmersa, observándose apoyo y compromiso por parte de su familia en cuanto al ámbito educacional y las demandas de este, ante lo cual su profesora jefe refiere: "la familia me apoya muchísimo a mí, no tengo nada que decir, es más, me apoya en cosas que no tiene que apoyarme, me prepara material excelente, prepara actividades, la apoyan mucho en lo educacional".

Desafíos:

La menor posee una escasa iniciativa, requiriendo de una persona de confianza que la estimule en este ámbito, lo que impacta en su autonomía y participación en actividades escolares, presentando además una baja tolerancia a la frustración: "el apoyo que se le entrega es personalizado, si no ella no termina algo y no tiene iniciativa de continuar con otra actividad y empieza a mirar al resto de sus compañeros, se pierde un poco".

En cuanto a la resolución de problemas dentro del contexto escolar, la menor es capaz de planificar soluciones, sin embargo, la mayor parte de éstas incluyen la participación de terceros, producto de la dificultad para ejecutar las soluciones de manera independiente. Su madre afirma: "reconoce el problema, pero prefiere pedir ayuda".

Los requerimientos producto de su condición, como: curaciones, controles médicos y tiempo destinado a las actividades de higiene y aseo, sumado a las constantes hospitalizaciones, generan una baja asistencia escolar; su madre afirma: "día por medio nos toca baño, eso me toma toda la mañana y después ella queda muy cansada... esos días no va al colegio... y en verano es peor, por las moscas". Esta situación de intermitente permanencia escolar provoca una baja identificación con el rol de estudiante, a pesar de que lo conoce y se involucra en la rutina y dinámica escolar; su profesora jefe refiere: "ha venido unas 10 veces en este año, pese a no tener dificultades para adaptarse a su rutina escolar, dificulta mucho sí, la identificación con el rol de estudiante".

Debido a las complicaciones de su condición física, C.S. no se desplaza de manera independiente, y se moviliza en silla de ruedas, acción que requiere asistencia de un tercero; su profesora jefe refiere: "ella ni siquiera tiene formados sus pies, tiene como muñoncitos, ya no se le formó el pie y cuando la trataron llevar a la Teletón sus dolores eran muy grandes, tiene como pegadita la parte de la rodilla atrás, no poder movilizarse y ser más independiente es una limitación tremenda". Cabe destacar, que su actual silla de ruedas no posee las características adecuadas para satisfacer las necesidades de la menor en su entorno físico, limitando su posibilidad de traslados. Las heridas y cicatrices en el rostro y cavidad oral dificultan tanto su comunicación verbal como no verbal, que sumado a su baja iniciativa restringen su habilidad para iniciar y mantener conversaciones, limitando así, su capacidad de relacionarse con sus compañeros; la profesora refiere: "tiene un desarrollo del lenguaje limitado, no vale la comparación pero al compararla con un niño de su edad ya debería por lo menos expresarse de forma más clara... utiliza poco el lenguaje, preguntas y respuestas es la forma en que ella se relaciona acá, realiza una conversación poco fluida y menos con los niños".

En cuanto al ambiente físico del establecimiento escolar, la accesibilidad es inadecuada para el desplazamiento de la usuaria; por otro lado, éste no presenta recursos físicos necesarios para sus requerimientos, ni adaptaciones que le permitan participar adecuadamente en las ocupaciones que el rol de estudiante exige. Sumado a esto, la comunidad escolar presenta una actitud de sobreprotección hacia C.S., limitando así su desarro- 
Ilo óptimo y su participación activa en este ambiente; en este sentido su profesora jefe refiere: "se conversó con los niños el caso de ella, había que tener muchísimo cuidado, fui muy aprensiva con ella porque me da pánico que le pase algo, que haya que curarla, que haya que hacer algo así con ella, nosotros no tenemos la preparación y menos en este tipo de patología... en los recreos muy pocas veces está sola, quizás nunca, de hecho los niños prefieren quedarse en la sala con ella y hay harta sobreprotección por parte de ellos". Respecto a este tema la usuaria refiere: "me gusta ir al colegio, aprendo a leer y escribir... no quiero salir en los recreos porque los niños más grandes me pueden pegar... me quedo en la sala con la tía".

\section{CASO 3}

\section{- Contexto}

C.H. es un joven de 14 años diagnosticado con E.B. distrófica, quien vive con sus padres y hermana menor, y reside en la comuna de Parral (VII Región). Asiste a una escuela rural desde hace 4 años, y cursa $4^{\circ}$ básico. Cabe destacar que no pudo incorporarse al sistema educacional con anterioridad, debido a que no contaba con el traslado necesario para asistir a la escuela.

\section{- Análisis del Perfil Ocupacional Fortalezas:}

En cuanto al aspecto volicional, C.H. posee las habilidades necesarias para seleccionar actividades de su preferencia dentro del establecimiento educacional, en las cuales participa activa y autónomamente, adecuándose a las demandas de las actividades tanto curriculares como extracurriculares; C.H. comenta: "para venir al colegio tengo que ir en bicicleta hasta la micro... en el recreo, hago lo que quiero, pero trato de que no sean juegos donde me pueda golpear, como las pilladas... a la salida me voy más tarde porque soy brigadista y ayudo a que los demás crucen la carretera".

En relación a las habilidades de comunicación e interacción C.H. posee una adecuada comprensión y expresión tanto verbal como no verbal, lo cual le permite adaptarse al interlocutor y mantener una conversación fluida y coherente. Cabe destacar que presenta una alteración en el volumen de la voz y en la articulación de las palabras, debido a las heridas y microstomía de la cavidad oral, lo cual no influye de manera significativa en su participación; su profesor refiere: "presenta un lenguaje más complejo que el que utiliza el resto de sus compañeros, usa a veces lenguaje que sus compañeros no entienden... su expresión no verbal es más lenta, su movimiento es más lento, le cuesta más".

Sus habilidades de procesamiento favorecen la participación ocupacional, puesto que le permiten cumplir con los objetivos académicos y desenvolverse adecuadamente en el ambiente escolar; "en cuanto a sus conocimientos se encuentra a la par con sus compañeros de curso, no tiene ninguna dificultad... durante las clases suele ayudar a sus compañeros a entender la materia a través de ejemplos que propone, es más maduro que ellos", comenta el profesor jefe.

El ambiente escolar posee recursos físicos que favorecen la participación ocupacional de C.H., quien se ha logrado adaptar a la rutina de establecimiento educacional, adoptando diversos roles, tales como alumno, amigo y brigadista del tránsito; en este sentido el profesor comenta: "nos preparamos, lo sentamos en una parte en la que los compañeros no lo molesten o se muevan mucho, entonces lo siento con mujeres, utilizamos también un cojín en el que se sienta en clases para evitar llagas, los materiales de la sala se encuentran a su alcance... su jornada escolar es igual a la del resto de sus compañeros... ha internalizado las rutinas diarias escolares y participa de ellas sin dificultad". Tanto el entorno familiar como escolar se encuentran sensibilizados con las características de C.H., lo cual favorece su inclusión a dicho contexto. Respecto de esto, la madre relata: "en el colegio lo apoyan mucho, siempre están preocupados por él, algunas veces hacen rifas y le compran de la leche que él necesita".

Desafíos:

La participación ocupacional de C.H. se ha visto afectada debido a que sus habilidades motoras se encuentran restringidas producto de las características de salud que presenta, tales como: menor desarrollo muscular, mayor gasto energético durante las actividades, escasa capacidad de realizar actividades que requieran prensiones finas y uso de ambas manos, producto de la pseudosindactilia que sólo le permite realizar pinza lateral; esto se suma a la permanencia del dolor producto de las heridas; "es más lento para escribir que sus compañeros, presenta dificultad para realizar trabajos manuales y sólo escribe con lápiz pasta... después del almuerzo se nota más cansado que el resto de sus compañeros, su mente no anda igual que su cuerpo", relata el profesor jefe. 
Cabe destacar que C.H. ingresó tardíamente al establecimiento educacional debido a la lejanía de éste en relación a su lugar de residencia y a la ausencia de alternativas de transporte. Por esta razón, es que actualmente se encuentra en $4^{\circ}$ básico y no en $1^{\circ}$ medio, a pesar de que sus capacidades son mayores. La madre refiere: "empezó a ir a la escuela hace poco, cómo 4 años, porque como vivimos lejos, antes no teníamos como llevarlo a la escuela... por el programa de movilización escolar ahora lo llevan, él sale hasta el camino en bicicleta y a veces caminando, ahí lo recoge un bus que lo trae en la tarde".

\section{Conclusiones}

A partir de los hallazgos obtenidos se evidencia que la manifestación de la E.B. en cada niño, sumado a su contexto, impacta de manera diferente en el desempeño y la participación ocupacional.

\section{Volición:}

Al analizar los tres casos, se evidencia una manera diferente en la forma que cada niño experimenta sus ocupaciones, debido a que se basan en la percepción de éxito o fracaso en las ocupaciones realizadas anteriormente y la aprobación o desaprobación que esto provoca en terceros, lo cual forma la autopercepción de capacidad, influenciando su participación en nuevas ocupaciones.

Los pensamientos y sentimientos volitivos están insertos en un ciclo en el cual intervienen la anticipación, la elección, las experiencias del "hacer" y su interpretación posterior (Kielfhofner, 2006). En general, algunas de las características de la E.B. han restringido la participación en la variedad de ocupaciones que brinda su ambiente, ya sea por experiencias insatisfactorias anteriores o por aprensiones de los padres o de la comunidad escolar. Los usuarios han visto influenciado su componente volicional debido a que la mayoría de ellos requiere de terceras personas de confianza para realizar o apoyar sus ocupaciones cotidianas, expresándose esto principalmente en una autopercepción de inseguridad o limitación de capacidades para un desempeño óptimo.

Es por esto, que los profesores jefes deben utilizar distintas estrategias e innovar para lograr que los niños y jó- venes con E.B. se involucren en las actividades tanto académicas como extra académicas. Estas estrategias deben tener un adecuado equilibrio entre las exigencias de la actividad y habilidades del menor, el nivel de participación, percepción de logro y motivación para involucrarse en ocupaciones futuras, en el ámbito escolar y otros. En este sentido, también se debe incluir la preparación y apoyo suficiente a los profesores para que puedan brindar las mejores oportunidades a estos niños, y de esta manera, logren desarrollarse integralmente.

Gracias a los hallazgos obtenidos a través de este estudio, podemos apreciar que, efectivamente, el rol del profesor jefe es fundamental a la hora de generar un nivel de motivación que le permita al niño involucrarse en las actividades escolares. En los tres casos, la relación con sus profesores jefes facilita este proceso, debido a que se utilizan estrategias como la graduación y modificación de algunas actividades, la planificación de actividades en las que los usuarios, a pesar de sus características físicas, puedan participar de manera activa junto a sus compañeros, fomentar la participación de la familia en el proceso de inclusión, entre otras.

\section{Habituación:}

Dado que, los niños con E.B. han debido adaptarse a diferentes rutinas preestablecidas a lo largo de su vida, tales como las hospitalarias, curación de heridas, de higiene y aseo, entre otras, el involucrarse en las rutinas del establecimiento educacional podría suponer un desafío posible, ya que a lo largo de su vida se han adaptado a diversos contextos estructurados, logrando desarrollar la capacidad de anticipar rutinas y participar activamente de ellas.

Esto se puede observar en el caso de C.S. quien, producto de sus constantes hospitalizaciones y procedimientos de curaciones diarios, le es fácil conocer, anticipar, adaptarse y participar activamente de sus rutinas. En los casos de V.V. y C.H. podemos observar una conducta similar, en la que a pesar de requerir ayuda física para realizar sus actividades, son autónomos en la mayoría de ellas.

Por otro lado, el asumir roles dentro del contexto escolar significa un gran desafío por la baja asistencia escolar, percepción negativa de sus habilidades, y a la escasa motivación en sus ocupaciones. Esta situación genera dificultades para el cumplimiento de las expectativas relacionadas con los roles en el ámbito escolar, en contraposición de una mayor identificación con ro- 
les relacionados a la condición de salud. En el caso de C.S. se puede apreciar baja asistencia escolar debido a largos períodos de hospitalizaciones, lo que genera una identificación con roles asociados a su enfermedad. El apoyo familiar y de la comunidad escolar, pueden impulsar al niño o joven a identificarse con su rol de estudiante, favoreciendo la inclusión al ambiente escolar. Esto se refleja en el caso de C.H., quien, producto de una buena gestión por parte de su profesor y su nivel de motivación, se ha involucrado en diferentes actividades dentro de la escuela, asumiendo el rol de estudiante, amigo y brigadista del tránsito, cumpliendo con las expectativas de estos y validándose a través de ellos.

\section{Habilidades de Comunicación e Interacción:}

Al analizar los tres casos se evidencia que esta área se ve interferida por las habilidades de comunicación verbal y la no verbal. La comunicación verbal se encuentra limitada frecuentemente por una microstomía de la cavidad oral, heridas y úlceras, que dificultan la articulación de las palabras, sumado a un tono de voz bajo. Con respecto a la comunicación no verbal se observa una disminución del potencial motor, que trae como consecuencia dificultades para moverse y gesticular.

En el contexto escolar estas limitaciones restringen la participación activa del estudiante en el aula de clases, tanto para solicitar asistencia de compañeros y profesores como hacer comentarios durante el transcurso de la jornada académica, al igual que en su interacción social con la comunidad escolar en actividades fuera de la sala de clases, tales como el recreo y actividades extra curriculares. Cabe destacar, que el lenguaje comprensivo no se encuentra alterado, ya que como se ha mencionado, no existe una alteración cognitiva asociada a la E.B.

\section{Habilidades de Procesamiento:}

Se sabe que en la E.B. no existe un deterioro de las habilidades cognitivas, sin embargo las habilidades de procesamiento se pueden ver afectadas por otras razones. En primer lugar, el aspecto volicional, que como se mencionó anteriormente, tiende a restringir la participación ocupacional de los usuarios, producto de la baja percepción de logro y, como consecuencia, una sensación de inseguridad. Requieren permanente ayuda de otros para desempeñarse, interfiriendo en el desarrollo de las estrategias personales necesarias para resolver problemas y abordar distintos desafíos.

Por otro lado, los usuarios suelen ingresar al sistema educacional tardíamente, debido principalmente a su condición de salud y cuidado, las dificultades para encontrar un establecimiento que los acepte, y las aprensiones de la familia. Una vez que el niño logra ingresar al sistema escolar, surgen las dificultades en torno a las exigencias del nivel y la adaptación del usuario que habitualmente se encuentra en un curso más bajo que el correspondiente según su edad. Se suma a este antecedente, el desconocimiento de la enfermedad por parte de la comunidad escolar. Muchas veces se le presentan exigencias cognitivas menores a los niños o jóvenes con E.B., restringiendo la posibilidad de desarrollar al máximo las capacidades que tenga.

En los tres casos se puede apreciar que las habilidades cognitivas se encuentran indemnes, sin embargo, los contextos escolares desarrollan de maneras distintas las capacidades en ellos, potenciándolas en algunos, a través de diferentes estrategias en las cuales se logra un equilibrio entre los desafíos y las capacidades del niño, a pesar de encontrarse en un nivel menor al que le corresponde; o limitándolas, al presentar desafíos menores a las capacidades cognitivas del estudiante como es el caso de V.V., quien asiste a una escuela especial donde no se realizan adaptaciones curriculares en relación a las actividades propuestas para sus compañeros.

Se puede concluir que la familia y los profesores que se involucran con los niños con E.B. cumplen un rol fundamental en el proceso de aprendizaje, la estimulación cognitiva y la participación social de estos niños y jóvenes.

\section{Habilidades Motoras:}

Como ya se mencionó, las personas con E.B. tienden a generar úlceras y ampollas en la piel y mucosas, que provocan una disminución de la movilidad general, producto del dolor y la cicatrización a la cual están expuestas a diario. Por otro lado presentan menor masa muscular y mayor gasto energético durante su participación en actividades cotidianas, dada la constante necesidad del cuerpo de reparar los tejidos dañados. El miedo a producir nuevas heridas y el dolor permanente durante la ejecución de las actividades, provoca una limitación en la participación tanto en el contexto familiar como escolar. 
Como consecuencia de las pseudosindactilias y manos en capullo, presentan dificultad en el uso de las manos, las prensiones finas y la coordinación bimanual, observándose principalmente en el uso de herramientas y objetos en el contexto escolar, tales como: tijeras, lápices y pinceles. En relación a las habilidades motoras gruesas presentan movimientos lentos, de menor amplitud y fluidez, que impacta en su participación en actividades que involucren mayor requerimiento y contacto físico.

Dado lo anterior, se infiere que los niños y jóvenes con E.B. pueden presentar limitaciones en su integración al contexto escolar si no se realizan las adaptaciones curriculares necesarias, modificación del ambiente físico, atención personalizada de profesionales y sensibilización del tema en la comunidad escolar. Las adaptaciones más frecuentes y requeridas por estos niños son: engrosar lápices y pinceles, acolchar sillas y mesas, rampas para el desplazamiento en silla de ruedas, incorporar medidas de seguridad en los juegos, discutir sobre la enfermedad y derribar prejuicios, entre otros.

\section{Ambiente:}

Para los niños con E.B. en sus tipos más severos, es fundamental desempeñarse en ambientes que les proporcionen seguridad y que reduzcan los riesgos de nuevas heridas o infecciones que puedan perjudicar su estado de salud. Cabe mencionar que los tres casos estudiados corresponden a usuarios que residen en sectores rurales, donde por lo general, el espacio físico restringe la participación de éstos, debido a la falta de infraestructura que permita un desplazamiento fácil y seguro.

Como se mencionó anteriormente, estos usuarios requieren de adaptaciones físicas para compensar déficit motores, con el objetivo de evitar posibles traumatismos y facilitar su desempeño. Debido al escaso presupuesto que suelen tener las escuelas rurales y la condición socioeconómica vulnerable de las tres familias, resulta complejo conseguir las adaptaciones necesarias para que los niños se desarrollen adecuadamente, potenciando sus capacidades y habilidades que les permitan incluirse de manera óptima al contexto escolar y a la comunidad en general.

En cuanto al ambiente social, éste influye de manera evidente en la participación ocupacional de estos usuarios. La familia tiende a sobreproteger a los menores, restringiendo sus capacidades físicas, cognitivas y de comunicación, por lo que, al enfrentarse a un contexto como el escolar, se ven muchas veces sobrepasados por una comunidad que no se siente preparada o no sabe cómo incluirlos.

Por otra parte, se evidencia que el ambiente escolar rural, no se encuentra diseñado y preparado para recibir a alumnos(as) con NEE en el ámbito físico, tendiendo a sobreproteger y limitar la cantidad de actividades que el niño puede hacer dentro y fuera del establecimiento. Esto trae como consecuencia, que no participe de manera activa en las instancias de interacción social como recreos, clases de educación física, talleres extracurriculares, entre otros, lo cual limita evidentemente la inclusión del niño y joven en el contexto escolar.

Con respecto a la participación ocupacional en el área de educación, se puede concluir que ésta es insatisfactoria, debido a que a pesar de formar parte de un establecimiento escolar, es decir estar integrados al sistema educacional, no se encuentran incluidos en éste, ya que dichos establecimientos desconocen las características de la enfermedad y no cuentan con los recursos físicos y humanos necesarios para identificar y eliminar los obstáculos a los que se ven enfrentados los niños, con el objetivo de lograr una igualdad de oportunidades y plena participación en dicho contexto.

\section{AgRADECIMIENTOS:}

Los más sinceros agradecimientos a aquellos usuarios, sus familias e instituciones educacionales que en forma voluntaria participaron del estudio, así como también al apoyo del equipo profesional de la fundación DEBRA Chile.

\section{RefERENCIAS BibLIOGRÁFICAS:}

Ares, L. (2012). Una evaluación ocupacional: marco de trabajo para la práctica de Terapia Ocupacional de la asociación americana de Terapia Ocupacional: dominio y proceso. Revista de Terapia Ocupacional de Galicia, 9 3-16.

Blesedell, E.; Boyt, B. y Cohn, E. (2005). Terapia Ocupacional, Teoría y Práctica en Terapia Ocupacional $\left(10^{a}\right.$ ed. pp. 477519).Madrid, España: Editorial médica panamericana.

Castillo, E.; López, H.; Fernández, R. y Cordovés, M. (2009). Epidermólisis Bullosa: una dermatosis poco conocida. Revista de ciencias médicas de Pinar del Río, 13, 71-82. 
Fine, J. (2010). Life with epidemolysis bullosa (EB) (3 ${ }^{\text {a }}$ ed.). Nueva York, Estados Unidos: Editorial Springer.

Kielhofner, G. (2006). Modelo de ocupación humana, Fundamentos conceptuales de la Terapia Ocupacional ( $3^{\text {a }}$ ed., pp. 147163). Santiago, Chile: Editorial médica panamericana.

Rojas, X. (2008). Reflexiones sobre la construcción de la percepción de exclusión social en jóvenes de enseñanza media: Precisiones conceptuales y metodológicas. Revista Última Década, 29, 71-99.

Solaque, H. y Vergara, E. (2009). Pseudosindactilia en epidermólisis bullosa. Revista de la facultad de medicina de la Universidad Nacional de Colombia, 57 275-280.

Yubero, M.; Kramer, S.; Gana, M.J.; Schwartz, R. y Palisson, F. (2010). Memoria anual 2010. Santiago, Chile: Debra Chile. 